\title{
Direct visualization of pancreatic intraductal papillary mucinous neoplasms in a patient with acute cholangitis
}

\author{
Chi Hyuk Oh
}

Division of Gastroenterology and Hepatology, Department of Internal Medicine, Kyung Hee University Hospital, Seoul, Korea
Received: August 7, 2020

Revised : August 19, 2020

Accepted: August 19, 2020

\section{Correspondence to}

Chi Hyuk Oh, M.D.

Tel: +82-2-958-8149

Fax: +82-2-958-8146

E-mail: ochihyuk@gmail.com https://orcid.org/0000-0002$4382-5876$
A 70-year-old male presented with acute recurrent cholangitis previously treated with endoscopic retrograde cholangiopancreatography (ERCP) at our hospital 5 years before. Approximately 10 years before, he underwent cholecystectomy for gallstone disease. Computed tomography revealed several stones within the dilatated common bile duct (CBD) and main pancreatic duct (MPD) dilatation, approximately $10 \mathrm{~mm}$ in diameter without intraductal mass or stones (Fig. 1A). Magnetic resonance cholangiopancreatography detected the MPD dilatation but no intraductal mass or stone (Fig. 1B). However, endoscopic ultrasonography revealed a tumorous lesion, approximately $8 \mathrm{~mm}$ in size, in the MPD (Fig. ${ }_{1 C)}$. ERCP was performed to remove the CBD stones and examine the MPD. Duodenoscopy revealed a fish-mouth appearance of the pancreatic opening (Fig. 1D). After CBD stone removal, direct pancreatoscopy (SpyGlass DS, Boston Scientific, Marlborough, MA, USA) was performed to identify the cause of MPD dilatation. SpyGlass demonstrated a mucin-producing papillary tumor in the MPD of the pancreatic head (Fig. 1E). Although the diffuse MPD dilatation with scar change expanded to the tail, no other tumorous lesion was observed within the MPD (Fig. 1F). Biopsy was performed using specially designed forceps for the SpyGlass (SpyBite). Pathology revealed intraductal papillary mucinous neoplasms (IPMNs). In accordance with the decision of the multidisciplinary team, the patient underwent a pylorus-preserving pancreaticoduodenectomy. The final pathological findings demonstrated IPMNs with intermediate-to-high grade dysplasia, no lymph node involvement, and negative resection margin (Fig. 2).

IPMNs, as in our patient, especially with a main duct IPMN accompanying high-risk features, has a significant potential of malignancy. Nevertheless, it may be misdiagnosed in a patient showing only dilatated MPD without any mass on imaging. Successful management of IPMNs relies on early detection and exact localization. Our case demonstrates the usefulness of direct pancreatoscopy using SpyGlass for exact diagnosis and proper management of IPMNs.

Written informed consent was obtained from the patient.

\section{Conflict of interest}

No potential conflict of interest relevant to this article was reported. 

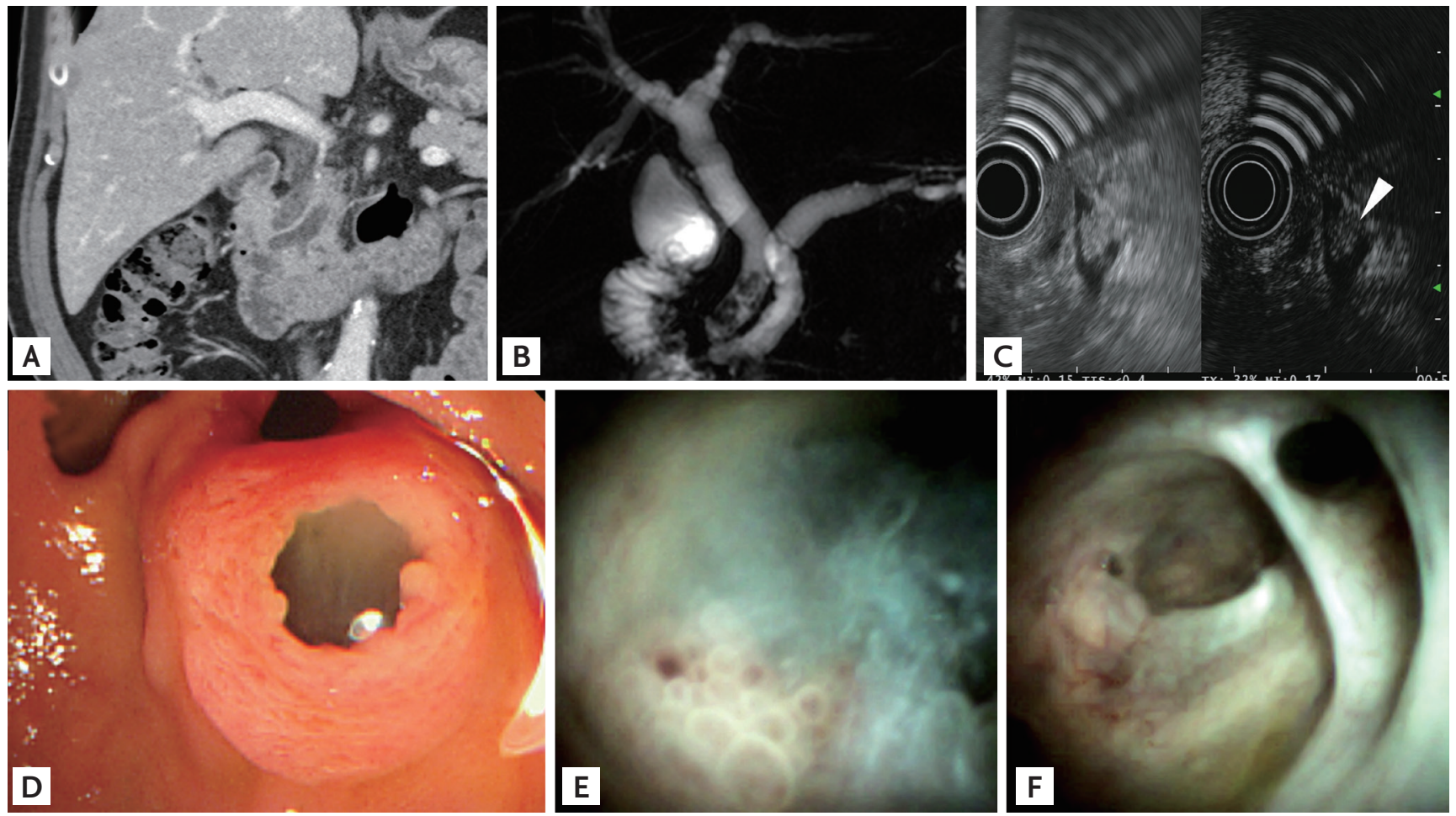

Figure 1. Imaging test for intraductal papillary mucinous neoplasms. (A) Computed tomography image showing only main pancreatic duct dilatation (MPD) with no mass/tumor. (B) Magnetic resonance cholangiopancreatography image showing common bile duct stones and MPD without intraductal mass. (C) Contrast-enhanced endoscopic ultrasonography image showing an enhancing mass (arrowhead) in the dilated MPD. (D) Duodenoscopy image showing a fish-mouth appearance of the pancreatic opening. (E) SpyGlass pancreatoscopy image showing a mucin-producing papillary tumor. (F) SpyGlass pancreastoscopy image showing a diffuse dilatated MPD with a scar change.
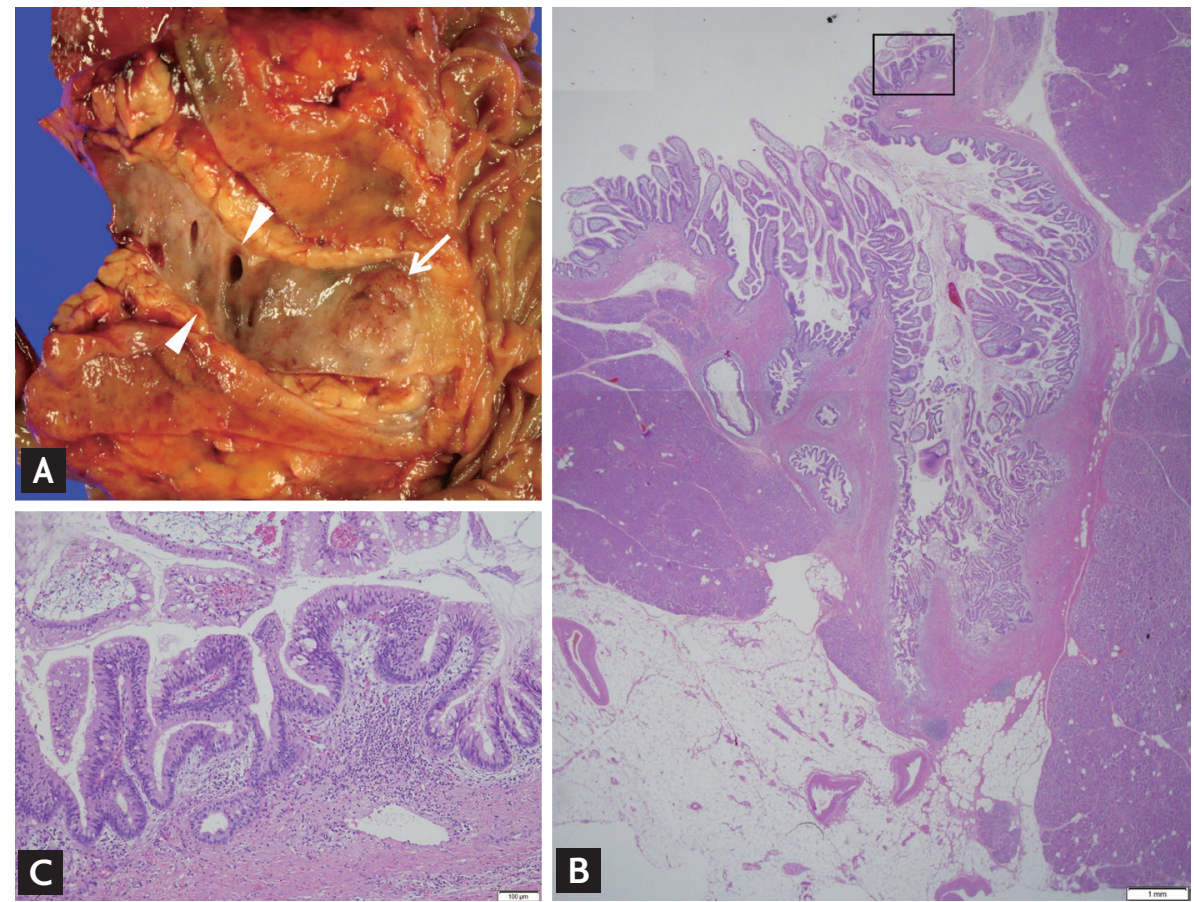

Figure 2. Pathological finding of intraductal papillary mucinous neoplasms (IPMNs). (A) Macroscopic finding from a specimen (arrowheads: dilatated main pancreatic duct; arrow: tumor). (B, C) Microscopic finding of IPMN $(\mathrm{H} \& \mathrm{E}, \times 4 \mathrm{O}$, black square in $\mathrm{B}: \mathrm{C}, \times 400)$. 